\title{
Mycobaterial Interspersed Repetitive Units-Variable Number of Tandem Repeats Analysis and Pattern of Drug Resistance in Extended Drug Resistant TB Isolates from Pulmonary Tuberculosis Patients in Bangladesh
}

\author{
Tarafder $\mathrm{S}^{1 *}$, Monir BB ${ }^{2}$ \\ ${ }^{I}$ Department of Microbiology and Immunology, Bangabandhu Sheikh Mujib Medical University, \\ Dhaka, Bangladesh; ${ }^{2}$ Residential Medical Officer, National institute of Laboratory Medicine and \\ Referral Center, Dhaka, Bangladesh.
}

\begin{abstract}
Background: Tuberculosis including multidrug resistance is a major public health problem in Bangladesh. Result of an advance treatment outcome of multidrug resistance is extended drug resistance tuberculosis.

Objective: This study was intended to observe the genotypes of XDR mycobacterium tuberculosis by determining 24 loci MIRU-VNTR analysis.

Methods: A total of 98 multidrug resistant tuberculosis (MDR-TB) isolates collected through Xpert MTB/RIF assay. They were subjected to $2^{\text {nd }}$ line (Fluoroquinolones, kanamycin, capreomycin and amikacin) drug susceptibility testing through line probe assay (LPA) in a view to detect extensively drug resistant tuberculosis (XDR-TB). Genotyping was done for XDR-TB isolates using 24 loci Mycobacterial Interspersed Repetitive Units-Variable Number of Tandem Repeats (MIRU-VNTR) using the online tool at www.MIRU-VNTRplus.org.. Out of 98 MDR-TB isolates 11(11.23\%) XDR-TB isolates were typed and analysed.

Results: Twenty four loci MIRU-VNTR genotyping involving similarity searching and phylogenetic tree analysis revealed that six $(54.6 \%)$ XDR-TB isolates belonged to Beijing strain, other MTB strain also detected were Delhi/CAS two(18.2\%), Haarlem two (18.2\%) and New-1, one $(9.1 \%)$ in number. Minimum spanning tree analysis showed two strain of Beijing family form a clonal complex. Beijing strains were more common among younger age group and within urban population. Beijing strains were also predominant in treatment failure patient. Only one new case of XDR-TB belongs to Delhi/CAS family. Second line mycobacterial drug resistance (MTBDRsl) detected by LPA showed the most prevalent mutations involved in fluoroquinolones drug resistance (FQ) was Asp94Gly in gyrA gene $(54.55 \%)$ in quinolone resistance determining region (QRDR) and for injectable $2^{\text {nd }}$ line drug resistance (ISL) was A1401G, C1402T in $16 \mathrm{~S}$ rrs gene (100\%).. All XDR-TB isolates showed resistance to levofloxacin in $2^{\text {nd }}$ line LPA but moxifloxacin showed low level resistance to some cases.
\end{abstract}

Conclusion: Molecular typing of XDR- TB isolates and pattern of drug resistance associated mutations in XDR-TB isolates in Bangladesh have not been reported previously. The result of this study highlights the need to reinforce the TB policy in Bangladesh with regard to control the spread and transmission as well as detection and treatment strategies regarding XDR-TB.

Keywords: MTB strains, XDR-TB, MIRU-VNTR Typing, Line probe assay

\section{Introduction}

Tuberculosis (TB) is a major public health problem in Bangladesh. The estimated number of all forms (new and relapse) TB cases were 221 per 100000 population in 2017. An estimated 36 per 100000 people died of TB in the same year. The incidence of Multi drug resistant (MDR-TB) was 5.1/100,000 population. Distribution of MDR-TB was $1.6 \%$ in new cases of TB and $29 \%$ in previously treated cases of TB in $2017 .^{1}$

*Correspondence: Dr. Shirin Tarafder, Department of Microbiology and Immunology, Bangabandhu Sheikh Mujib Medical University;

e-mail: starafder2007@yahoo.com; ORCID: 0000-0002-1632-6828
Extensively drug resistant tuberculosis (XDR-TB) is defined as TB resistant to Rifampicin (RMP) and Isoniazid (INH) with additional resistance to second line anti-TB drugs i.e. to any fluoroquinolones (FQs), and to at least one of the three injectable second-line drugs (ISL) naming amikacin (AMK), kanamycin (KAN) and capreomycin (CAP). ${ }^{2}$ The prevalence of XDRTB is $8.5 \%$ worldwide. ${ }^{1}$ XDR-TB is the result of an adverse treatment outcome of MDR-TB; many cases are never diagnosed due to limitations in laboratory capacity to test for second-line drug resistance. ${ }^{3}$ Treatment of XDR-TB patients is more challenging and less successful than that of 
patients with other types of TB. ${ }^{4} \mathrm{An}$ extremely high death rate from XDR-TB was reported in patients co-infected with HIV in South Africa. ${ }^{5}$

Molecular epidemiological studies of $\mathrm{M}$. tuberculosis identified variability in the phylogeography of strains globally. ${ }^{6}$ Beijing strains are most prevalent globally and also associated with enhanced acquisition of drug resistance; however their resistance patterns varied regionally. ${ }^{7}$

There are information of 186 different types of strains are present at online databases. These strains are well characterized for genotypic analysis. The commonly found strains worldwide are Beijing, Cameroon, Delhi/Central Asian (CAS), East African-Indian (EAI), Ghana, Haarlem, Latin American-Mediterranean (LAM), Uganda I and II, Ural, and X lineages. ${ }^{8}$

The types of strains vary country to country as well as regionally. EAI, the Delhi and the Beijing genotypes are the major strains in India, LAM in Brazil and CAS in Kenya. ${ }^{9-11}$ There are several typing methods that are used to detect strains of MTB. Among them IS6110 based RFLP, MIRUVNTR, Spoligotyping, Single nucleotide polymorphisms (SNPs), Deletion analysis, Whole genome sequencing (WGS), technologies are widely used. MIRU-VNTR typing has become an important method as it allows high throughput, discriminatory and reproducible analysis of clinical isolates. ${ }^{8,12}$ 24-loci mycobacteria interspersed repetitive units-variable number of tandem repeat (MIRU-VNTR) can provide unique high-resolution insights into the population structure of the MTB and provides clear criteria for the identification of the different MTB lineages and sub-lineages. ${ }^{8,12}$ Now MIRU-VNTR becomes newly recommended gold standard for phylogenetic analysis of MTB. ${ }^{13,14}$

The World Health Organization (WHO) recommended the use of molecular line probe assay (LPA) for rapid screening of MDR-TB, PreXDR-TB and XDR-TB.LPA use multiplex PCR amplification and hybridization to identify $M$ tuberculosis complex and mutation to genes associated with different drug resistance. ${ }^{1}$ Second Line LPA shows good specificity and sensitivity for the detection of FLQ resistance ( $\geq 86 \%$ and $\geq 99 \%$ ) and second line injectable drug (SLID) resistance $(\geq 87 \%$ and $\geq 99 \%)$ compared to phenotypic drug sensitivity testing on culture isolate or directly sputum specimen. ${ }^{15}$
Although the prevalence of XDR-TB alarmingly increases in Bangladesh, till now to the best of knowledge, no published data on mutations and genotypes of XDR-MTB strains are currently available in Bangladesh.

To gain an insight about molecular typing of MTB, 24 loci MIRU-VNTR has been performed in this study. Drug resistance-associated mutations in XDR-TB isolates from Bangladesh has also been documented by using $2^{\text {nd }}$ line LPA (MTBDRsl).

\section{Materials and Methods}

Study period and place: This cross sectional study was conducted from March 2018 to February 2019 at the Department of Microbiology and Immunology, Bangabandhu Sheikh Mujib Medical University (BSMMU) and National Tuberculosis Reference Laboratory (NTRL) at National Institute of Diseases of the Chest and Hospital (NIDCH) Dhaka, Bangladesh.

Study population: The ethical clearance of the research project was obtained from the BMRC National Research Ethics Committee. Purposive sampling procedure was followed. Samples were collected after taking informed written consent of the patient and data were collected in a predesigned data collection sheet. Sample size was determined considering the prevelance rate of XDR-TB in India. ${ }^{16}$ Total 98 Pulmonary MDR-TB patients diagnosed by Xpert MTB/RIF assay were selected in this study. Drug sensitive tuberculosis patients were excluded from the study. Microscopy, DNA extraction and second line LPA was done in NTRL and 11 XDR-TB were identified. 24 loci MIRUVNTR typing of 11 XDR-TB isolates were performed at Department of Microbiology and Immunology, BSMMU.

Procedures: The confirmed MDR-TB sample isolates were collected at NTRL. These isolates were cultured on both Löwenstein - Jensen media and mycobacterial growth indicator tube (MGIT). DNA extraction was performed following standard operating procedure ${ }^{12}$. Extracted DNA was transferred to BSMMU in vials within cold box to perform conventional PCR for MIRUVNTR analysis.

24 loci MIRU-VNTR typing and Analysis: MIRUVNTR typing was based on amplification by PCR. It could be performed on heat inactivated mycobacterial colonies or mycobacterial pellets from liquid cultures without extensive DNA purification. ${ }^{12}$ Qiagen Master Mix kit including Q solution and primers were used for amplification. Amplification was carried out in an automated DNA 
thermal cycler (Applied bio-system 2720). PCR cycle run as follows: $15 \mathrm{~min}$ at $95^{\circ} \mathrm{C}, 1 \mathrm{~min}$ at $94^{\circ} \mathrm{C}$, $30 \mathrm{~s}$ at $59^{\circ} \mathrm{C}, 1 \mathrm{~min} 30 \mathrm{~s}$ at $72^{\circ} \mathrm{C}$ (these were repeated for 40 cycles), $10 \mathrm{~min}$ at $72^{\circ} \mathrm{C}$, and $\alpha$ at $4^{\circ} \mathrm{C}$. The amplified product was detected by Gel electrophoresis was done in 3\% agarose for 5 hours at 120 constant voltage. Gel was stained with ethidium bromide to detect the specific DNA bands. Marker of DNA of known bp (100 bp ladder) was used to determine the size of amplified PCR products. The DNA bands of the samples were visualized by using a UV trans-illuminator and photograph was taken for further documentation.

Strain identification: There was a standard allelic table which was found from the database website. The number of repeats for each locus was determined based on this allelic table. Ultimately a numerical datum was found for 24 loci for every isolates based on this allelic table. The numerical data were exported to the reference data base. MIRU-VNTR plus web tools were used for identification and comparison of strains by similarity search and phylogenetic tree calculation.

Molecular drug susceptibility testing by line probe assay: Genotype MTBDRsl assay was performed according to the instructions provided by the manufacturer (Hain Life science GMbH,
Nehren, Germany) for second line LPA. ${ }^{17}$ The extracted DNA was amplified by using HAIN amplification master mix. Amplification at PCR and Hybridization of the amplified sample were performed according to the instructions. There were both mutation probes and wild type probes for each gene. The presence of any mutation band regarded as resistance to the respective antibiotic. Absence of at least one of the wild type was also regarded as resistant.

Statistical analysis: The number of repeats for each locus was determined based on the allelic table ${ }^{12}$ and clustering analysis was done using the online tool at http://www.MIRU-VNTRplus.org. ${ }^{12}$

\section{Results}

24 loci MIRU-VNTR typing: Among 11 XDR-TB isolates 24 locus MIRU-VNTR typing revealed Beijing was the predominant strain six $(54.6 \%)$ in number, followed by Delhi/CAS, two (18.2\%) and Harlem two (18.2\%). Other strain was identified as NEW-1 one (9.10\%) (figure 1). Minimum spanning free analysis through MIRUVNTR plus web tool showed two of the Beijing strains formed a clonal complex. Rest of the nine isolates was remaining as Singletons. (figure 2)

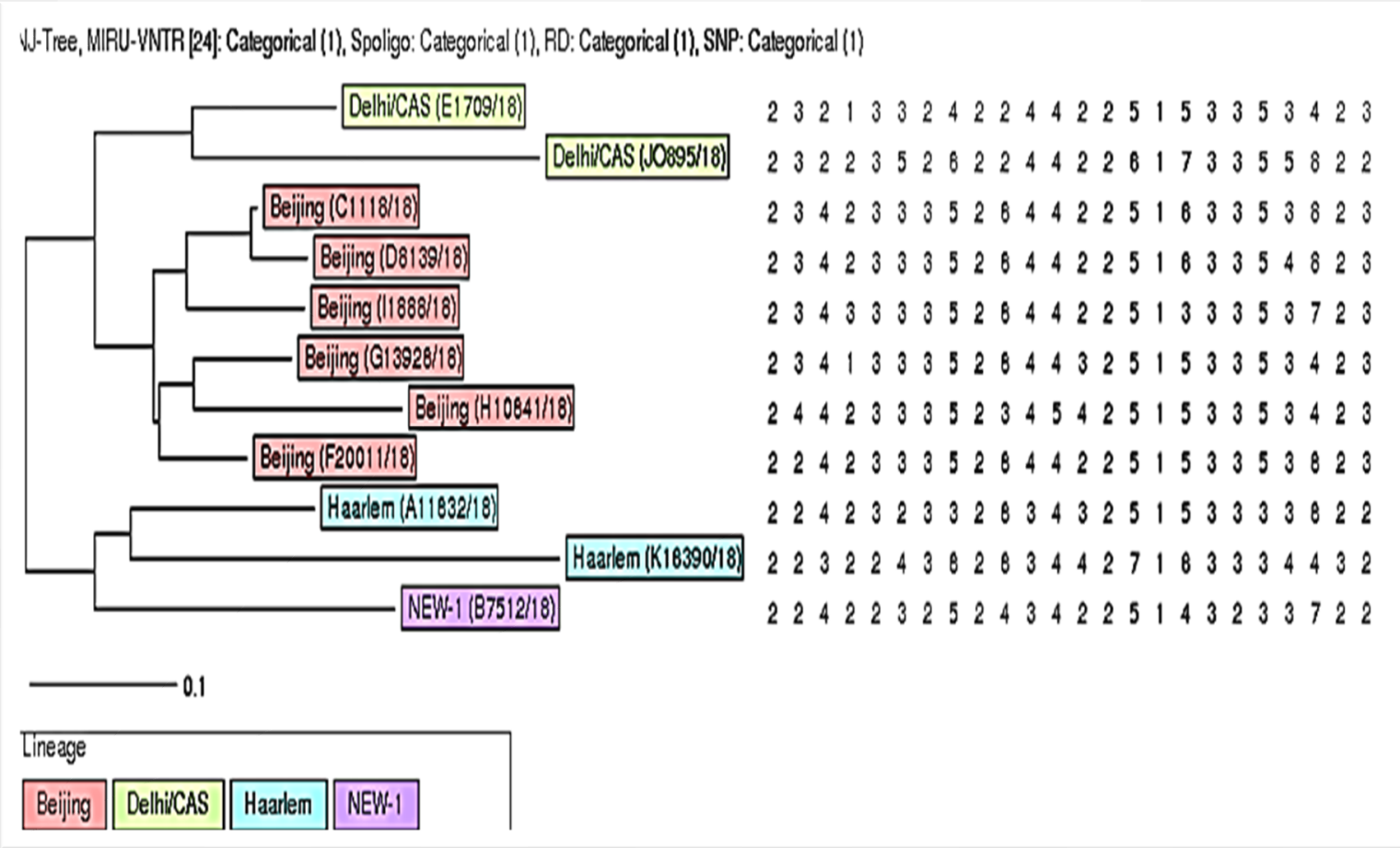

Figure 1: N-J (Near joining) tree of XDR-TB isolates generated through MIRU-VNTR web plus tool 


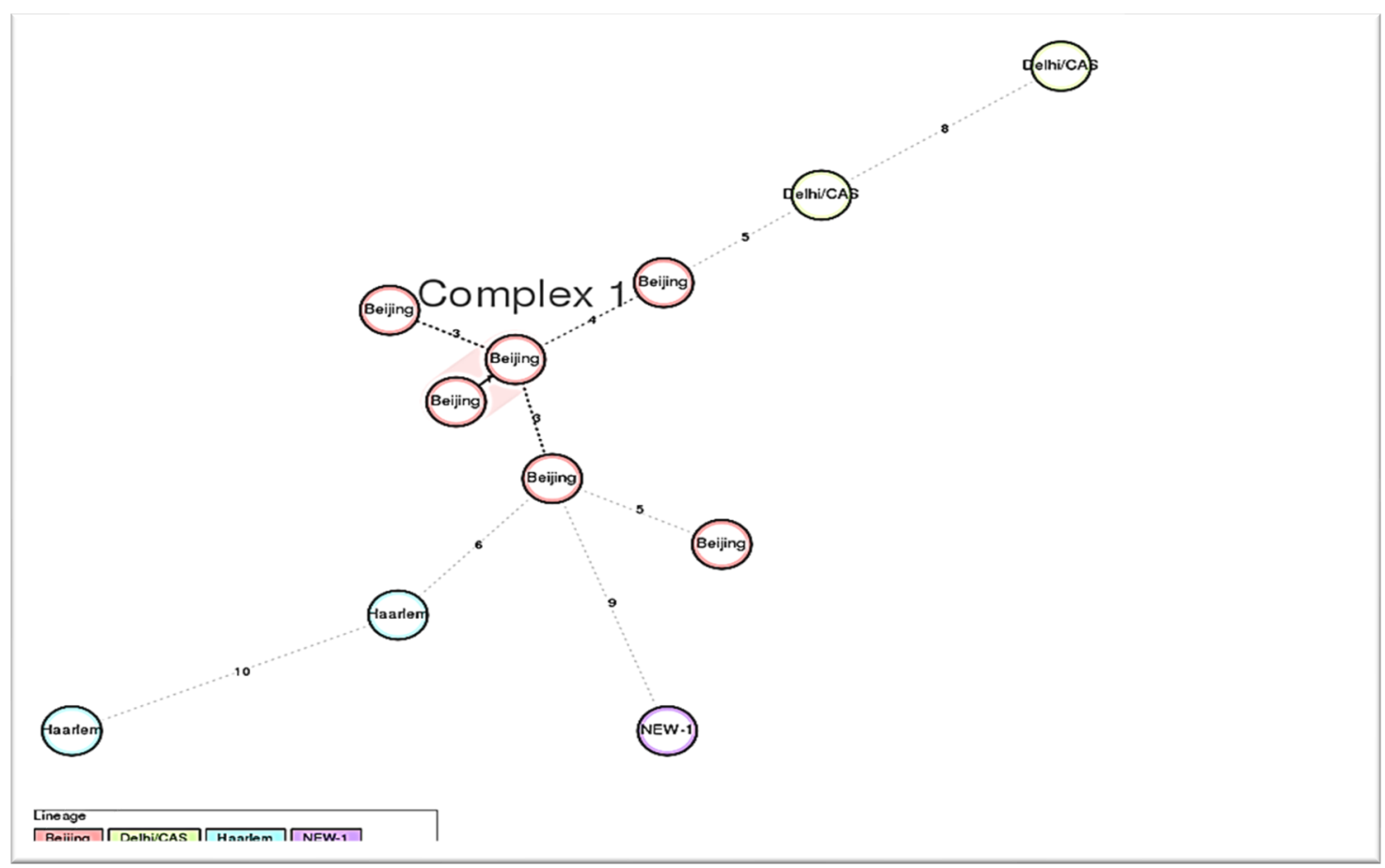

Figure 2: Minimum spanning tree of 11 XDR-TB isolates generated through MIRU-VNTR plus web tool.

*Clonal Complex: A clonal complex in this analysis was defined as a group of strains with no more than a single locus variation.

*Singletons: Strains not found in the clonal complexes regarded as Singletons.

Association of XDR-TB isolates with TB treatment outcome:

Among 11 XDR-TB patients, there are four (36.4\%) Cat 2 relapse cases. MIRU-VNTR analysis shows among these four cases two cases belonged to Beijing genotype and one each for Delhi/CAS and Haarlem genotype. Four $(36.4 \%)$ Cat 2 failure cases turned into XDR-TB also and among them two were Beijing genotype and one each for Haarlem and NEW-1 genotype. Only one case $(9.1 \%)$ of Cat 1 relapse and one case $(9.1 \%)$ of Cat 1 non converter turned into XDR-TB, and both of them belonged to Beijing genotype. Newly diagnosed XDR-TB case was only $1(9.1 \%)$ in number that belonged to Delhi/CAS genotype. (figure 3)

Socio demographic data and risk factors associated with XDR-TB isolates:

Among 11 XDR-TB isolates seven cases were male $(63.6 \%)$ that were significantly higher than the four female $(36.4 \%)$ patients. Most patients were within 30-39 years of age group (45.5\%). Urban populations were greater in number (63.6 $\%)$ than the rural populations.

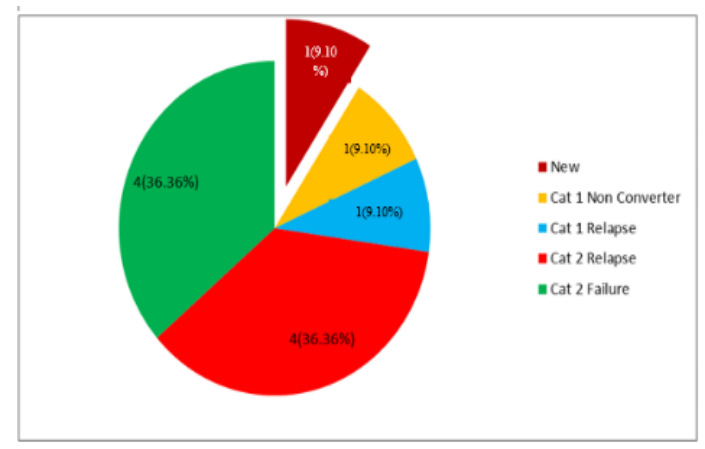

Figure 3: Association of XDR-TB isolates with TB treatment outcome.

Almost all are BCG vaccinated Ten (90.9\%). Most of the patient having no history of drug and alcohol misuse. Population characteristics among the Lineages also indicate six $(60.0 \%)$ cases out of ten of the previously treated patients with antiTB drugs were belonged to Beijing family(table I)

Drug resistance pattern: Second line LPA determines the resistance pattern of 11 XDR-TB isolates. Regarding fluoroquinolones (FQ) resistance among 11 XDR-TB isolates, six (54.55\%) showed point mutation at MUT 3C \{gyr A Asp94Gly (GAC-GGC)\}. Followed by three (27.27\%) XDR-TB isolates showed point 
Table I: Socio demographic data and risk factors associated with XDR-TB isolates

\begin{tabular}{lll} 
Categories & \multicolumn{1}{c}{ Sub categories } & $\begin{array}{l}\text { XDR } \\
\text { (n=11) }\end{array}$ \\
\hline Socio demographic Data & \\
Sex & Male & $7(63.64)$ \\
& Female & $4(36.36)$ \\
Age & $20-29$ & $2(18.18)$ \\
& $30-39$ & $5(45.45)$ \\
& $40-49$ & $3(27.27)$ \\
Location & $50-59$ & $1(9.10)$ \\
& Urban & $7(63.64)$ \\
Occupation & Rural & $4(36.36)$ \\
& Service & $1(9.10)$ \\
& Business & $5(45.45)$ \\
BCG vaccine mark & Housewife & $4(36.36)$ \\
& Self-employed & $1(9.10)$ \\
Risk factors & Present & $10(90.90)$ \\
H/O TB contact & Absent & $1(9.10)$ \\
& & \\
H/O Anti-TB treatment & Yes & $1(9.10)$ \\
Smoking & No & $10(90.90)$ \\
& Previously Treated & $1(9.10)$ \\
Other illness & Yes & $7(60.90)$ \\
& No & $4(36.36)$ \\
& Yes & $2(18.18)$ \\
& No & $9(81.82)$ \\
\hline
\end{tabular}

mutation at MUT 3B \{gyr A Asp94Asn (GACAAC) Asp94Tyr (GAC-TAC)\}. Rest of two $(18.18 \%) \quad$ XDR-TB isolates showed point mutation at MUT 1\{ gyr A Ala90Val (GCGGTG)\}. None had mutations in the quinolone resistance determining region (QRDR) of $g y r \mathrm{~B}$. Regarding injectable $2^{\text {nd }}$ line drug resistance (ISL) all 11 XDR-TB isolates showed point mutation at MUT $1\{r r s(\mathrm{~A} 1401 \mathrm{G}),(\mathrm{C} 1402 \mathrm{~T})\}$. Beijing Lineage showed all pattern of mutation in gyr A six $(54.60 \%)$ but interestingly MUT1 was only seen in Beijing lineage two (18.20\%) whereas Haarlem lineage showed resistance only in gyr A MUT3C two $(18.20 \%)$ and NEW-1 lineage showed only mutation in MUT3B one $(9.10 \%)$. Delhi/CAS lineage showed point mutation in MUT3B $1(9.10 \%)$ and MUT3C one $(9.10 \%)$. There were no drug resistance showed in gyr B. All 11 XDRTB isolates showed resistance at $16 \mathrm{~S}$ rrs MUT 1 $11(100 \%)$. There were no drug resistance showed in $r r s$ MUT 2 and eis MUT 1(table II).

Table II: Correlation of Fluoroquinolones and Injectable second line drug resistance with MTB lineages among XDR-TB isolates $(\mathrm{n}=11)$

\begin{tabular}{|c|c|c|c|c|c|c|c|c|}
\hline \multirow[t]{2}{*}{ Drug resistance } & \multicolumn{2}{|l|}{$\begin{array}{l}\text { Wild type } \\
\text { band }\end{array}$} & \multirow[t]{2}{*}{$\begin{array}{l}\text { Developing } \\
\text { mutation band }\end{array}$} & \multicolumn{4}{|c|}{ Lineage } & \multirow[t]{2}{*}{$\begin{array}{l}\text { Total } \\
\text { No. }(\%) \\
\end{array}$} \\
\hline & Developed & $\begin{array}{l}\text { Not } \\
\text { developed }\end{array}$ & & $\begin{array}{l}\text { Beijing } \\
\text { No. }(\%)\end{array}$ & $\begin{array}{l}\text { Delhi/CAS } \\
\text { No. (\%) }\end{array}$ & $\begin{array}{l}\text { Haarlem } \\
\text { No. }(\%)\end{array}$ & $\begin{array}{l}\text { NEW-1 } \\
\text { No.(\%) }\end{array}$ & \\
\hline \multirow{9}{*}{ Fluoroquinolones } & \multirow{4}{*}{$\begin{array}{l}g y r \text { A } \\
\text { WT 1* }\end{array}$} & - & \multirow{4}{*}{$\begin{array}{l}\text { Not developed } \\
\text { MUT } 1^{* *} \\
\text { MUT 2 } \\
\text { MUT 3A }\end{array}$} & - & - & - & - & $0(0)$ \\
\hline & & gyr A & & $2(18.2)$ & $0(0)$ & $0(0)$ & $0(0)$ & $2(18.2)$ \\
\hline & & WT $2 * *$ & & $0(0)$ & $0(0)$ & $0(0)$ & $0(0)$ & $0(0)$ \\
\hline & & \multirow{3}{*}{$\begin{array}{l}g y r \text { A } \\
\text { WT } 3\end{array}$} & & $0(0)$ & $0(0)$ & $0(0)$ & $0(0)$ & $0(0)$ \\
\hline & \multirow{5}{*}{$g y r$ B WT } & & MUT 3B** & $1(9.1)$ & $1(9.1)$ & $0(0)$ & $1(9.1)$ & $3(27.3)$ \\
\hline & & & MUT $3 C^{* *}$ & $3(27.3)$ & $1(9.1)$ & $2(18.2)$ & $0(0)$ & $6(54.6)$ \\
\hline & & & MUT 3D & $0(0)$ & $0(0)$ & $0(0)$ & $0(0)$ & $0(0)$ \\
\hline & & & MUT 1 & $0(0)$ & $0(0)$ & $0(0)$ & $0(0)$ & $0(0)$ \\
\hline & & - & MUT 2 & $0(0)$ & $0(0)$ & $0(0)$ & $0(0)$ & $0(0)$ \\
\hline \multirow{6}{*}{$\begin{array}{c}\text { Injectable } 2^{\text {nd }} \text { line } \\
\text { Drug }\end{array}$} & $r r s$ & $r r s$ WT $1 * *$ & MUT $1 * *$ & $4(36.4)$ & $2(18.2)$ & $2(18.2)$ & $1(9.1)$ & $9(81.8)$ \\
\hline & $\mathrm{WT} 1 * * *$ & - & MUT $1 * * *$ & $2(18.2)$ & $0(0)$ & $0(0)$ & $0(0)$ & $2(18.2)$ \\
\hline & $r r s$ WT 2 & - & MUT 2 & $0(0)$ & $0(0)$ & $0(0)$ & $0(0)$ & $0(0)$ \\
\hline & eis WT 1 & - & Not developed & $0(0)$ & $0(0)$ & $0(0)$ & $0(0)$ & $0(0)$ \\
\hline & eis WT 2 & - & $\begin{array}{ll}\text { MUT 1 not } \\
\text { developed }\end{array}$ & $0(0)$ & $0(0)$ & $0(0)$ & $0(0)$ & $0(0)$ \\
\hline & eis WT 3 & - & Not developed & $0(0)$ & $0(0)$ & $0(0)$ & $0(0)$ & $0(0)$ \\
\hline
\end{tabular}

Note:

*The strain regarded as sensitive when all the wild type of bands were present and all the mutation type bands were absent.

**The presence of any mutation band regarded as resistance to the respective antibiotic. Absence of at least one of the wild type was also regarded as resistant.

***The presence of both mutation band and corresponding wild type band regarded as heteroresistant strain or presence of more than one M. tuberculosis complex strain in specimen.

\section{Discussion}

Beijing was the major XDR-TB strains followed by Delhi/CAS, Haarlem and NEW-1 in this study. A study in Nepal showed the predominance of Beijing strains (69.2\%) among XDR-TB isolates ${ }^{18}$.
A study in China revealed $83.1 \%$ belonged to the Beijing family. ${ }^{19}$ A study in Japan found Beijing $(62.0 \%)$ as the predominant lineage followed by $\mathrm{T}$ $(13.0 \%)$, LAM (5\%) and EAI $(2.0 \%){ }^{20}$

24 loci MIRU-VNTR analysis showed the predominance of Beijing strains. The similar 
involvement of Beijing genotypes in XDR-TB cases has been reported from South Africa, Japan India $^{21}$ and China. ${ }^{5,20,22}$ In this study predominance of Beijing genotype in XDR-TB cases supported the data of previous study that this genotype has been associated with drug resistance, because of its higher mutation rates and lower fitness costs with specific mutation. ${ }^{23}$

In Bangladesh, the higher number of MDR-TB patients who didn't completed the anti TB treatment could be responsible for this high drug resistance acquisition rate. Both the possibility of direct transmission and acquired resistance should be considered equally for XDR-TB in Bangladesh.

This study showed all 11 XDR-TB isolates had point mutation at gyr A of fluoroquinolones of which most of the point mutation were at codon Asp94Gly (MUT 3C) followerd by codon Asp94Tyr (MUT 3B) and codon A90V (MUT 1). In a study conducted in China revealed $80.0 \%$ mutation at codon 94 of gyrA gene in XDR-TB isolates. ${ }^{24}$ Different study in South Africa showed the majority of mutations were observed at codon 94 of gyrA gene. ${ }^{25,26}$ All XDR-TB isolates showed resistance to Levofloxacin in $2^{\text {nd }}$ line LPA but moxifloxacin showed low level resistance to some cases, so, moxifloxacin can be used at higher doses for treatment of those cases.

Eleven XDR-TB isolates showed point mutation at nucleotide 1401 and 1402 of $r r s$ gene of injecctable second line drugs. Similar finding also found in a study in China. ${ }^{24}$ Different studies conducted in South Africa showed a predominant rrs gene mutation at nucleotide 1401 of injectable second line drugs. ${ }^{25,26}$

In this study, among Cat 2 relapse cases and Cat 2 failure cases, most of them belonged to Beijing genotype followed by Delhi/CAS and Haarlem. Notably Cat 2 failure cases, Cat 1 relapse case and Cat 1 non converter cases turned into XDR-TB, all of them belonged to Beijing genotype. Newly diagnosed XDR-TB case belonged to Delhi/CAS genotype. Regarding association of MTB strains with TB treatment outcome there are no available data to compare with this study.

Beijing lineage was prevalent among treatment failure and relapse cases. The exact mechanism of the treatment failure by Beijing lineage is not well known. It is hypothesised that the basal mutation rate of this lineage is higher than the other. ${ }^{27}$

\section{Conclusion}

The data of this study provide important baseline information on the genetic diversity of $M$. tuberculosis among XDR-TB cases in Bangladesh. Therefore, it could be used to monitor changes in the transmission pattern of tuberculosis. Findings emphasise the urgent need to identify the patients suffering from XDR-TB with Beijing genotype and to treat them as early as possible. It also suggests to conduct epidemiological survey by using genotyping methods in order to provide adequate data that can be used for the formulation of a better control programme to prevent the spread.

Conflict of interests: The authors declare that there is no conflict of interests.

Funding: The study was supported by the Bangladesh Medical Research Council, Dhaka.

Submitted: $19^{\text {th }}$ September, 2019

Final revision received: $12^{\text {th }}$ March, 2020

Accepted: $19^{\text {th }}$ March, 2020

Published online: $1^{\text {st }}$ April, 2020

\section{Reference}

1. Global tuberculosis report 2018. Geneva: World Health Organization. 2018.

2. Banerjee R, Allen J, Westenhouse J, Elms W, Desmond E, Nitta A et al. Extensively drugresistant tuberculosis in California, 1993-2006. Clinical Infectious Diseases. 2008; 47:450-57.

3. Shah NS, Richardson J, Moodley P, Moodley S, Babaria P, Ramtahal $\mathrm{M}$ et al. Increasing drug resistance in extensively drug-resistant tuberculosis, South Africa. Emerging infectious diseases. 2011; 17:510.

4. Banerjee R, Schecter GF, Flood J, Porco TC. Extensively drug-resistant tuberculosis: new strains, new challenges. Expert review of antiinfective therapy. 2008; 6:713-24.

5. Mlambo CK, Warren RM, Poswa X, Victor TC, Duse AG, Marais E. Genotypic diversity of extensively drug-resistant tuberculosis (XDR-TB) in South Africa. The international journal of tuberculosis and lung disease. 2008; 12:99-104.

6. Filliol I, Driscoll JR, Van Soolingen D, Kreiswirth $\mathrm{BN}$, Kremer K, Valétudie G et al. Global distribution of Mycobacterium tuberculosis spoligotypes. Emerging Infectious Diseases. 2002; 8:1347.

7. Glynn JR, Whiteley J, Bifani PJ, Kremer K, van Soolingen D. Worldwide occurrence of Beijing/W strains of Mycobacterium tuberculosis: a systematic review. Emerging Infectious Diseases. 2002; 8:843. 
8. Allix-Béguec C, Harmsen D, Weniger T, Supply $\mathrm{P}$, Niemann S. Evaluation and strategy for use of MIRU-VNTRplus, a multifunctional database for online analysis of genotyping data and phylogenetic identification of Mycobacterium tuberculosis complex isolates. Journal of Clinical Microbiology. 2008; 46:2692-99.

9. Gutierrez MC, Ahmed N, Willery E, Narayanan S, Hasnain SE, Supply $\mathrm{P}$ et al. Predominance of ancestral lineages of Mycobacterium tuberculosis in India. Emerging Infectious Diseases. 2006; $12: 1367$.

10. Oelemann MC, Gomes HM, Willery E, Lima KV, Allix-Béguec C, Supply P et al. The forest behind the tree: phylogenetic exploration of a dominant Mycobacterium tuberculosis strain lineage from a high tuberculosis burden country. PLoS One. 2011; 6:e18256.

11. Ndungu PW, Kariuki S, Revathi G, Niemann S. Mycobacteria Interspersed Repetitive UnitsVariable Number of Tandem Repeat, Spoligotyping and Drug Resistance of Isolates from Pulmonary Tuberculosois Patients in Kenya. Advances in Microbiology. 2017; 7:205.

12. Supply, P., Allix, C., Lesjean, S., CardosoOelemann, M., Rüsch-Gerdes, S., Willery, E et al.Proposal for standardization of optimized mycobacterial interspersed repetitive unitvariable-number tandem repeat typing of Mycobacterium tuberculosis. Journal of Clinical Microbiology. 2006; 44:4498-4510.

13. Desikan S, Narayanan S. Genetic markers, genotyping methods \& next generation sequencing in Mycobacterium tuberculosis. The Indian Journal of Medical Research. 2015; 141:761.

14. Jagielski T, Van Ingen J, Rastogi N, Dziadek J, Mazur PK, Bielecki J. Current methods in the molecular typing of Mycobacterium tuberculosis and other mycobacteria. BioMed Research International. 2014

15. World Health Organization. The use of molecular line probe assays for the detection of resistance to second-line anti-tuberculosis drugs: policy guidance. World Health Organization. 2016.

16. Singhal P, Dixit P, Singh P, Jaiswall, Singh M, Jain A. A study on pre-XDR \& XDR tuberculosis \& their prevalent genotypes in clinical isolates of Mycobacterium tuberculosis in north India. The Indian Journal of Medical Research. 2016 ; 143: 341.

17. Hain Lifescience $\mathrm{GmbH}$, Nehren, Germany. GenoType MTBDRsl, url: www.hain-lifescience. de/en/instructions-foruse.html.
18. Poudel A, Maharjan B, Nakajima C, Fukushima $\mathrm{Y}$, Pandey BD, Suzuki Y et al. Characterization of extensively drug-resistant Mycobacterium tuberculosis in Nepal. Tuberculosis. 2013; 93: 84-88.

19. $\mathrm{Hu} \mathrm{Y}$, Hoffner $\mathrm{S}, \mathrm{Wu} \mathrm{L}$, Zhao Q, Jiang W, Xu B. Prevalence and genetic characterization of secondline drug-resistant and extensively drug-resistant Mycobacterium tuberculosis in Rural China. Antimicrobial agents and chemotherapy. 2013; 57:3857-63.

20. Murase $\mathrm{Y}$, Maeda S, Yamada H, Ohkado A, Chikamatsu K, Mizuno K et al. Clonal expansion of multidrug-resistant and extensively drugresistant tuberculosis, Japan. Emerging Infectious Diseases. 2010; 16:948.

21. Ajbani K, Rodrigues C, Shenai S, Mehta A. Mutation detection and accurate diagnosis of extensively drug-resistant tuberculosis: report from a tertiary care center in India. Journal of Clinical Microbiology. 2011; 49:1588-90.

22. Sun Z, Chao Y, Zhang X, Zhang J, Li Y, Qiu Yet al. characterization of extensively drug-resistant Mycobacterium tuberculosis clinical isolates in China. Journal of Clinical Microbiology. 2008; 46:4075-77.

23. Gagneux S. Fitness cost of drug resistance in Mycobacterium tuberculosis. Clinical Microbiology and Infection. 2009; 15:66-68.

24. $\mathrm{Hu} \mathrm{Y}$, Hoffner S, Wu L, Zhao Q, Jiang W, Xu B. Prevalence and genetic characterization of secondline drug-resistant and extensively drug-resistant Mycobacterium tuberculosis in Rural China. Antimicrobial Agents and Chemotherapy. 2013;57:3857-63.

25. Gardee Y, Dreyer AW, Koornhof HJ, Omar SV, Da Silva P, Ismail NAet al. Evaluation of the GenoTypeMTBDRsl version 2.0 assay for secondline drug resistance detection of Mycobacterium tuberculosis isolates in South Africa. Journal of Clinical Microbiology. 2017; 55:791-800.

26. Said HM, Kock MM, Ismail NA, Baba K, Omar $\mathrm{SV}$, Osman AG et al. Evaluation of the GenoType ${ }^{\circledR}$ MTBDRsl assay for susceptibility testing of second-line anti-tuberculosis drugs. The International Journal of Tuberculosis and Lung Disease. 2012;16:104-09.

27. Ford CB, Shah RR, Maeda MK, Gagneux S, Murray MB, Cohen $\mathrm{T}$ et al. Mycobacterium tuberculosis mutation rate estimates from different lineages predict substantial differences in the emergence of drug-resistant tuberculosis. Nature Genetics. 2013; 45:784. 